\title{
Direct evidence of superconductivity and determination of the superfluid density in buried ultrathin $\mathrm{FeSe}$ grown on $\mathrm{SrTiO}_{3}$
}

\author{
P. K. Biswas, ${ }^{1,}{ }^{*}$ Z. Salman, ${ }^{1}$ Q. Song, ${ }^{2}$ R. Peng,,${ }^{2}$ J. Zhang, ${ }^{2}$ L. Shu, ${ }^{2}$ D. L. Feng, ${ }^{2}$ T. Prokscha, ${ }^{1}$ and E. Morenzoni ${ }^{1, \dagger}$ \\ ${ }^{1}$ Laboratory for Muon Spin Spectroscopy, Paul Scherrer Institut, CH-5232 Villigen PSI, Switzerland \\ ${ }^{2}$ State Key Laboratory of Surface Physics, Department of Physics, Fudan University, Shanghai 200433, China
}

(Received 18 December 2017; published 11 May 2018)

\begin{abstract}
Bulk FeSe is superconducting with a critical temperature $T_{c} \cong 8 \mathrm{~K}$ and $\mathrm{SrTiO}_{3}$ is insulating in nature, yet hightemperature superconductivity has been reported at the interface between a single-layer FeSe and $\mathrm{SrTiO}_{3}$. Angleresolved photoemission spectroscopy and scanning tunneling microscopy measurements observe a gap opening at the Fermi surface below $\approx 60 \mathrm{~K}$. Elucidating the microscopic properties and understanding the pairing mechanism of single-layer FeSe is of utmost importance as it is a basic building block of iron-based superconductors. Here, we use the low-energy muon spin rotation/relaxation technique to detect and quantify the supercarrier density and determine the gap symmetry in $\mathrm{FeSe}$ grown on $\mathrm{SrTiO}_{3}(100)$. Measurements in applied field show a temperature-dependent broadening of the field distribution below $\sim 60 \mathrm{~K}$, reflecting the superconducting transition and formation of a vortex state. Zero-field measurements rule out the presence of magnetism of static or fluctuating origin. From the inhomogeneous field distribution, we determine an effective sheet supercarrier density $n_{s}^{2 \mathrm{D}} \simeq$ $6 \times 10^{14} \mathrm{~cm}^{-2}$ at $T \rightarrow 0 \mathrm{~K}$, which is a factor of 4 larger than expected from ARPES measurements of the excess electron count per Fe of 1 monolayer FeSe. The temperature dependence of the superfluid density $n_{s}(T)$ can be well described down to $\sim 10 \mathrm{~K}$ by simple $s$-wave BCS, indicating a rather clean superconducting phase with a gap of 10.2(1.1) meV. The result is a clear indication of the gradual formation of a two-dimensional vortex lattice existing over the entire large FeSe/STO interface and provides unambiguous evidence for robust superconductivity below $60 \mathrm{~K}$ in ultrathin FeSe.
\end{abstract}

DOI: 10.1103/PhysRevB.97.174509

\section{INTRODUCTION}

Following the discovery of high- $T_{c}$ cuprates $[1,2]$ a few decades ago, the Fe-based superconductors [3-8] represented an additional novel and important class of high- $T_{c}$ superconductors displaying, however, average critical temperatures lower than the cuprates. Surprisingly, high-temperature superconductivity with a $T_{c} \approx 60-70 \mathrm{~K}$ was found in single-layer FeSe on $\mathrm{SrTiO}_{3}$ (STO) [9-13]. Similar high temperatures exceeding that of all known bulk iron-based superconductors have also been achieved on other oxide substrates [14].

This finding is extremely important in view of the simplecrystal structure of the system, which consists of a single Se-FeSe unit, i.e., the basic building block of all iron-chalcogenide superconductors, and may pave the way to identifying key ingredients of high- $T_{c}$ superconductivity [10]. Single-layer FeSe exhibits a distinct electronic structure with only electron pockets near the Brillouin zone corner [11-13]. This is in contrast to its bulk counterpart, which also shows hole pockets at the zone center.

Transport measurements performed ex situ find, with respect to bulk, an enhancement of $T_{c}$ with onset around 40 $\mathrm{K}$ not only in $1 \mathrm{ML}$ FeSe [15], but also in ultrathin layers

*Current address: ISIS Pulsed Neutron and Muon Source, STFC Rutherford Appleton Laboratory, Harwell Campus, Didcot, Oxfordshire, OX11 0QX, UK; pabitra.biswas@stfc.ac.uk

†elvezio.morenzoni@psi.ch in various configurations [16] including electric-double-layer transistor films [17] and ultrathin flakes on $\mathrm{SiO}_{2} / \mathrm{Si}$ [18]. Similar $T_{c}$ as on STO have been measured on other substrate materials such as $\mathrm{MgO}, \mathrm{KTaO}_{3}$ [19], $\mathrm{TiO}_{2}$ (rutile [14] and anatase phase [20]), and K-doped FeSe films [21], whereas in situ zero resistivity was detected at a temperature as high as $109 \mathrm{~K}$ [22]. Diamagnetic shielding was also observed up to $T_{\text {onset }} \sim 65 \mathrm{~K}[23]$

The superconducting gap of FeSe/STO has been mainly characterized by surface-sensitive techniques such as ARPES and STM. The data suggest that single-layer FeSe has plain $s$-wave pairing symmetry $[10,11,13,24]$. However, on its own, detection of a gap appearing below $\sim 60 \mathrm{~K}$ does not provide conclusive evidence that it is only related to the formation of a condensate of Cooper pairs and does not exclude other contributions such as magnetic, charge, or spin density wave gaps. Transport measurements, on the other hand, cannot easily discriminate between filamentary and bulk superconductivity. It is therefore essential to characterize the presence of superconductivity in $\mathrm{FeSe} / \mathrm{STO}$ and its microscopic properties by other techniques, providing complementary information such as the superfluid density and the homogeneity of the superconducting phase.

Here, we report detailed depth-resolved investigation of the superconducting and magnetic properties in ultrathin $\mathrm{FeSe}$ by the low-energy muon spin rotation/relaxation (LE- $\mu \mathrm{SR})$ technique. Zero-field (ZF) $\mu \mathrm{SR}$ measurements demonstrate that the ground state is nonmagnetic and transverse-field (TF) $\mu$ SR results show that superconductivity appears below $62 \mathrm{~K}$. 


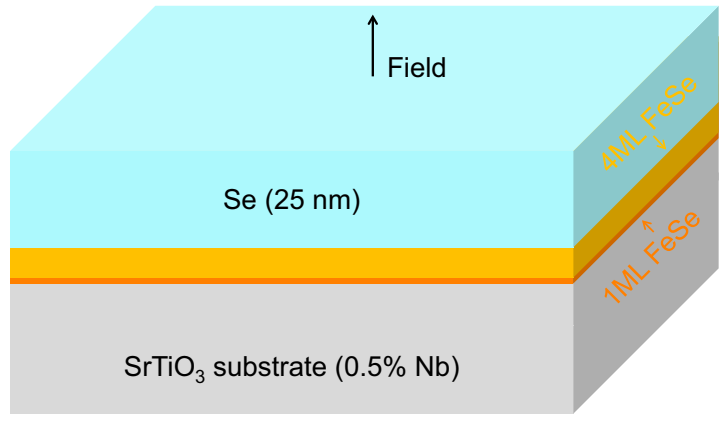

FIG. 1. Layers of the heterostructure. Schematic diagram (not to scale) of the heterostructure with a ultrathin FeSe film grown on the $\mathrm{SrTiO}_{3}$ substrate. For transverse-field measurements, the magnetic field is applied perpendicular to the sample surface. The polarization of the implanted muons is parallel to the sample surface.

Taking into account the extreme two-dimensional character of the vortex state, we estimate the effective superfluid sheet density $n_{s}^{2 \mathrm{D}}(T)$. Its temperature dependence is well described down to $\sim 10 \mathrm{~K}$ by a simple BCS $s$-wave model, with a gap $\Delta(0)=10.2(1.1) \mathrm{meV}$.

\section{EXPERIMENTAL DETAILS}

\section{A. Film growth and characterization}

Figure 1 shows a schematic of the heterostructure used in this experiment. Single-layer FeSe thin films were grown using molecular beam epitaxy (MBE) on a $10 \times 10 \mathrm{~mm}^{2} \mathrm{TiO}_{2}$ terminated and $\mathrm{Nb}$-doped (0.5\% wt.) (001)-oriented $\mathrm{SrTiO}_{3}$ substrate. The substrate was precleaned following the method described in previous work [13] and ultrahigh vacuum (UHV) condition was maintained during deposition to enable continuous in situ growth. In the UHV chamber, the substrate was degassed at $550{ }^{\circ} \mathrm{C}$ for $3 \mathrm{~h}$ and then heated to $9500^{\circ} \mathrm{C}$ under a $\mathrm{Se}(99.9999 \%)$ flux for $30 \mathrm{~min}$. It was kept at $490^{\circ} \mathrm{C}$ in Se and Fe $(99.995 \%)$ flux for coevaporation and codeposition with the flux ratio of 20:1. After growth, the films were annealed at $600{ }^{\circ} \mathrm{C}$ in vacuum for $3 \mathrm{~h}$. In situ measurements confirmed the possible $60-\mathrm{K}$ superconductivity in the monolayer (ML) FeSe film. Four more unit cells of FeSe thin films were successfully grown above the single-layer FeSe. The additional layers were deposited for stabilization purpose since, surprisingly, the original tunneling spectra of two unit cells or thicker FeSe films did not show signs of superconductivity [9]. Before depositing the overlayers, the FeSe ML was characterized by ARPES. Figure 2 shows the result exhibiting the typical features of the electronic structure [11,13]. ARPES measurements indicated charge transfer from the substrate and superconductivity to be restricted to the FeSe interface layer with the top layer displaying charge neutrality. However, in our discussion below we will also address the question of the possible contribution of these additional layers to the observed supercarrier density, in view of our and recent results of charge distributions in ultrathin films [19]. A $\sim 25 \mathrm{~nm}$ thick layer of amorphous Se was added for protection. Thickness of the films was monitored using a crystal oscillator and confirmed by x-ray reflectivity measurements. A susceptibility measurement by mutual induction on a sample of similar composition and
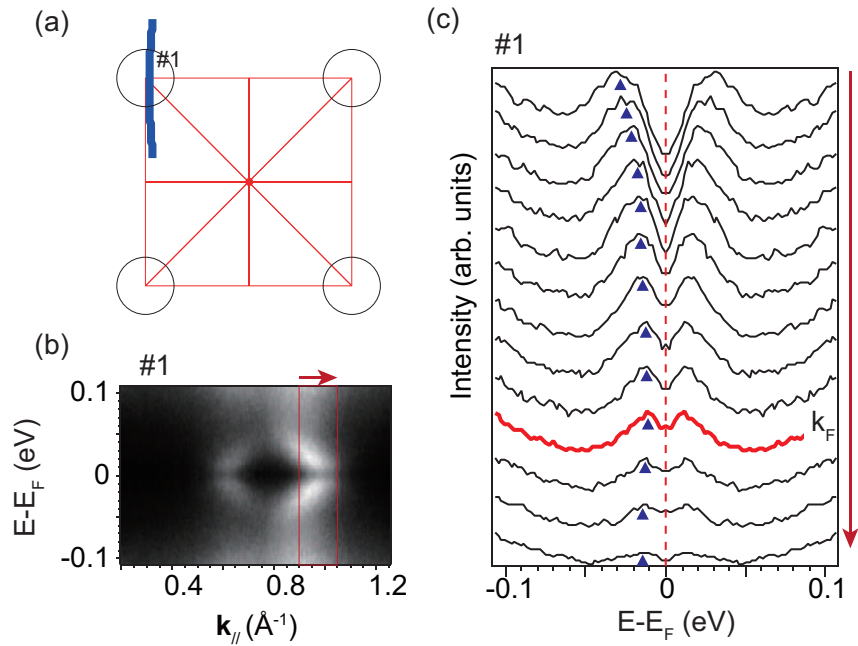

FIG. 2. ARPES measurements. (a) Sketch of the Fermi surface sheets and Brillouin zone of single-layer FeSe/STO. Cut \#1 is indicated in the Brillouin zone. (b) The photoemission intensity along cut \#1, which is symmetrized with respect to Fermi energy. (c) Symmetrized energy distribution curves along a portion of cut \#1 which is indicated by the red arrow in panel (b). Data were collected at $25 \mathrm{~K}$.

structure, grown under the same condition and equipment as the sample presented here, provided unambiguous evidence for the onset of Meissner effect at $65 \mathrm{~K}$ [23]. The $\mu$ SR measurements reported here were performed on a mosaic of three pieces of the $10 \times 10 \mathrm{~mm}^{2}$ surface area films. The samples were glued to a Ni-coated Al plate and mounted onto a cold finger cryostat. Ni suppresses the $\mu$ SR signal from the muons not hitting the sample [25].

\section{B. Low-energy $\mu \mathrm{SR}$}

To measure the local magnetic and superconducting properties of the ultrathin FeSe layer, we use LE- $\mu$ SR as a sensitive magnetic probe [26]. Fully polarized muons are implanted in the sample one at a time, where they thermalize and act as sensitive magnetic microprobe. The muon spin precesses around the local magnetic field $B$ at the muon site with the Larmor frequency $\omega_{\mu}=\gamma_{\mu} B, \frac{\gamma_{\mu}}{2 \pi}=135.5 \mathrm{MHz} / \mathrm{T}$. The precession and relaxation of the spin ensemble leads to a temporal evolution of the polarization, which is easily detectable via the asymmetric muon decay (lifetime $\tau_{\mu}=2.2 \mu \mathrm{s}$ ), where a positron is emitted preferentially in the direction of the muon spin at the moment of the decay. From the damped precession signal the field distribution associated with the vortex state can be determined. The LE- $\mu$ SR experiments were performed on the LEM instrument, at the $\mu \mathrm{E} 4$ beamline of the Paul Scherrer Institut in Villigen, Switzerland [27]. Here, the energy of the muons can be tuned $(\sim 1$ to $30 \mathrm{keV})$ to control the implantation depth in the range $(\sim 1-300) \mathrm{nm}$ and thus to probe the magnetic response in different layers of the heterostructure [28]. With this unique ability, the LE- $\mu$ SR technique is an ideal probe for studying the superconducting properties of the FeSe layer by implanting the muons on or very close to this layer. This procedure has been successfully applied to address 


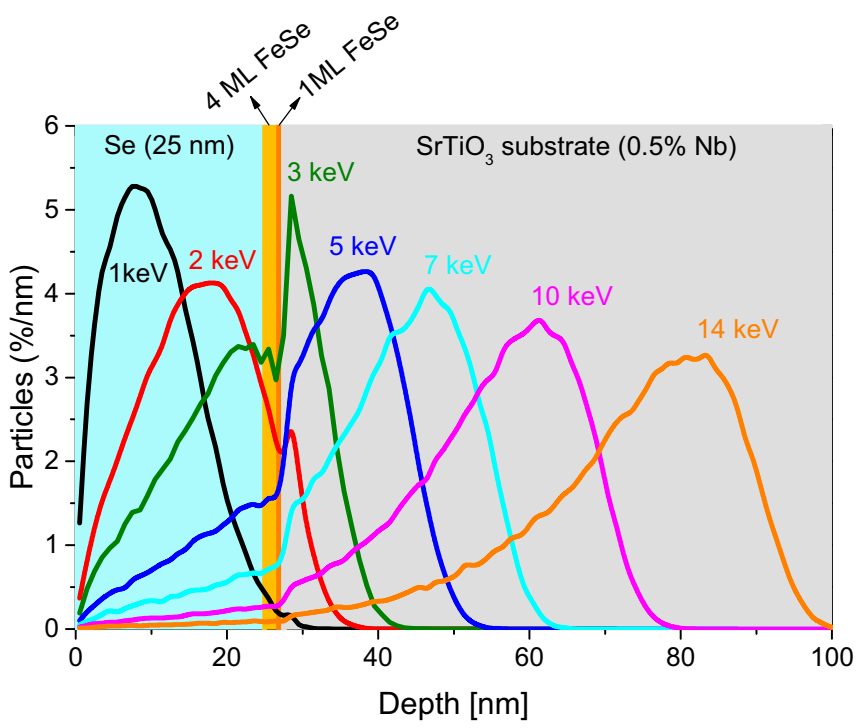

FIG. 3. Muon implantation profiles. Muon stopping profiles in the investigated heterostructure calculated at different implantation energies using the Monte Carlo code TRIM.SP modified for muon implantation.

related questions in a variety of systems and heterostructures. In particular, by varying the implantation energy of the muons, the spatial evolution of the magnetic field distribution as the flux lines emerge through the surface of a superconducting $\mathrm{YBa}_{2} \mathrm{Cu}_{3} \mathrm{O}_{7-\delta}$ film has been monitored [29], superconducting proximity effects of buried cuprate layers [30], the paramagnetic Meissner effect due to spin-triplet components [31], and magnetism at transition-metal-molecular interfaces have been detected [32].

\section{Zero-field and transverse-field $\mu$ SR measurements}

Initially, we tuned the muon beam implantation energy $E$ to maximize the fraction of muons stopping in the vicinity of the FeSe single layer. Monte Carlo simulations, presented in Fig. 3, show that this is achieved for $E \sim 3 \mathrm{keV}$. The program TRIM.SP, especially modified for muon implantation in heterostructures and whose reliability to calculate stopping profiles has been previously tested, was used for the calculation [33,34].

We performed $\mathrm{ZF}$ and TF- $\mu \mathrm{SR}$ measurements at different temperatures. A ZF measurement is very sensitive to magnetism; in a magnetic environment, well-defined precession frequencies may be observed in the case of long-range order. Alternatively, a distribution of precession frequencies with the corresponding width proportional to the field inhomogeneity may be detected. If the field distribution is broad when averaged over the sample, as in the case of disordered or short-range magnetism, the muon decay asymmetry displays a fast depolarization. In the case of dynamic moments with fluctuating times within the $\mu$ SR time window, spin relaxation is also observed. These features allow the direct observation of the onset of magnetic order even if very weak. It has been used for instance to search for time-reversal symmetry-breaking phenomena in the superconducting phase, where a very tiny spontaneous static magnetic field appears with the onset of superconductivity $[35,36]$. The ZF spectra taken at $2.3 \mathrm{keV}$

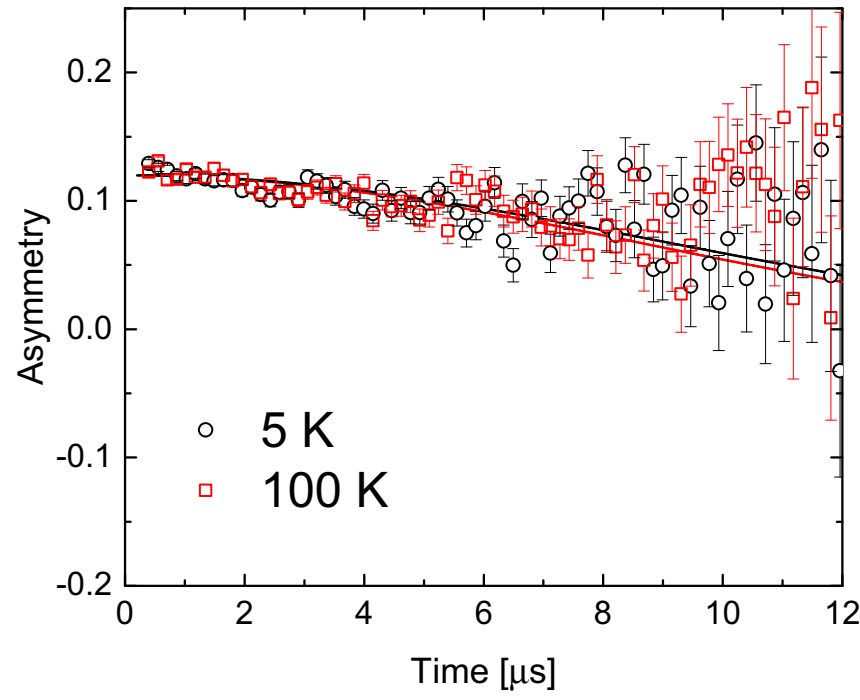

FIG. 4. ZF muon spin relaxation. ZF- $\mu$ SR time spectra collected at 5 and $100 \mathrm{~K}$ for single-layer FeSe with muon implanted at an energy of $2.3 \mathrm{keV}$. The solid lines are fits to the data. See text for details about the fit function.

muon implantation energy can be described well using a static Gaussian Kubo-Toyabe relaxation function [37], where the time evolution of the asymmetry $A(t)$, which is proportional to the muon spin polarization, is given by

$$
A(t)=A_{0}\left\{\frac{1}{3}+\frac{2}{3}\left(1-\sigma_{\mathrm{ZF}}^{2} t^{2}\right) \exp \left(-\frac{\sigma_{\mathrm{ZF}}^{2} t^{2}}{2}\right)\right\},
$$

where $A_{0}$ is the initial asymmetry and $\sigma_{\mathrm{ZF}}$ the muon spin relaxation rate. We do not detect any difference in the spectra, taken at 5 and $100 \mathrm{~K}$, as shown in Fig. 4. The nearly equal and very small values of $\sigma_{\mathrm{ZF}}\left[0.086(5)\right.$ and $0.082(5) \mu \mathrm{s}^{-1}$ for 5 and $100 \mathrm{~K}$, respectively], extracted from the fits for two different temperatures, reflect the presence of random local magnetic fields arising solely from the nuclear moments in the sample.

For the TF- $\mu$ SR measurements as a function of temperature, the sample was cooled in a magnetic field of $10 \mathrm{mT}$ applied normal to the sample surface and to the initial muon spin direction.

Figure 5shows the TF- $\mu$ SR time spectra collected at (a) $5 \mathrm{~K}$ and (b) $70 \mathrm{~K}$. At $70 \mathrm{~K}$, the local field probed by the muons corresponds to the applied field and only a weak damping of the signal is observable, consistent with the ZF results at $100 \mathrm{~K}$. By contrast, the data collected at $5 \mathrm{~K}$ show a more pronounced damping. The $\mu$ SR time spectra (Fig. 5) were analyzed using a Gaussian damped spin precession signal [38]:

$$
A(t)=A_{0} \exp \left(-\sigma^{2} t^{2} / 2\right) \cos \left(\gamma_{\mu} B t+\phi\right),
$$

where $A(0)$ is the initial asymmetry, $B$ is the magnetic field at the muon sites, $\phi$ is the initial phase of the muon polarization precession signal, and $\sigma(T)$ is the spin damping rate due to the field inhomogeneities. 


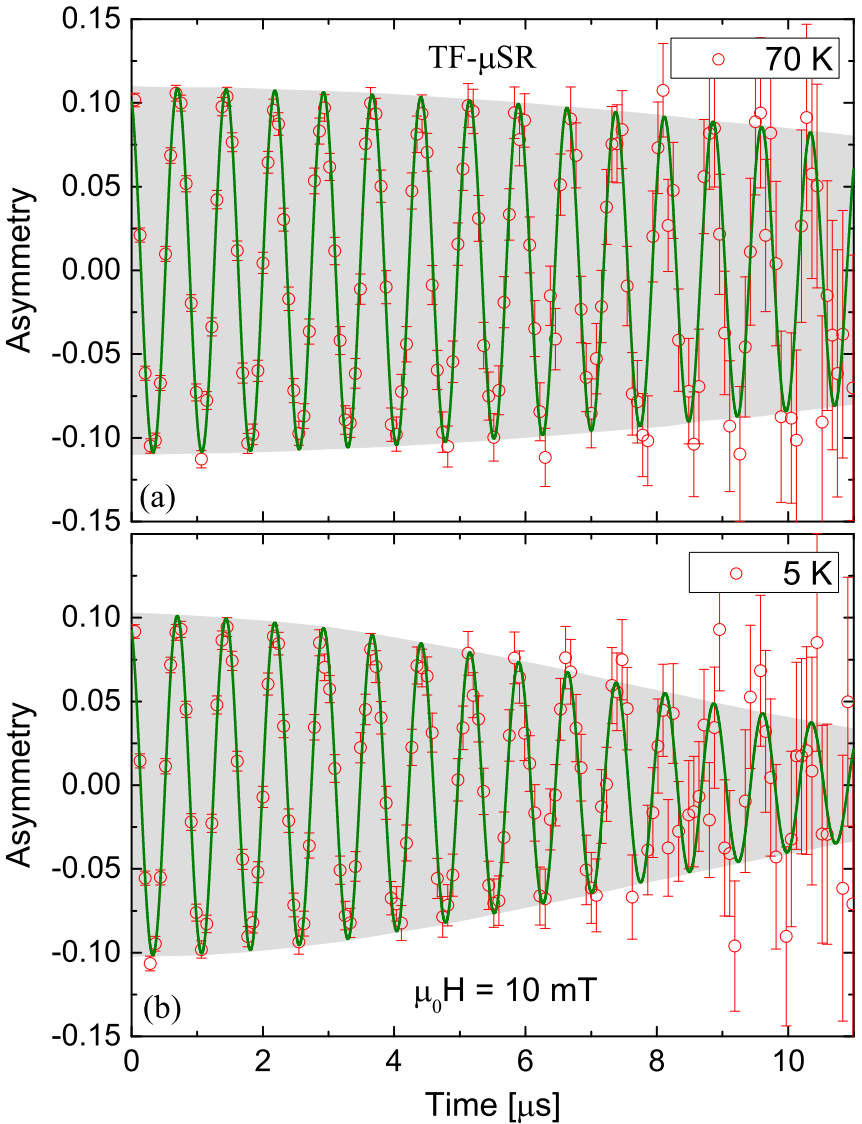

FIG. 5. Muon spin rotation signal. TF- $\mu$ SR time spectra collected in a transverse field of $10 \mathrm{mT}$ with a muon implantation energy of $3 \mathrm{keV}$ at temperatures (a) $5 \mathrm{~K}$ and (b) $70 \mathrm{~K}$. The solid lines are fits to the data using Eq. (2). The shaded area evidences the different damping rate.

\section{RESULTS AND DISCUSSION}

\section{A. Temperature and energy dependence of the field broadening}

The temperature dependence of the Gaussian damping rate $\sigma(T)=\left[\sigma_{\mathrm{sc}}^{2}(T)+\sigma_{\mathrm{nm}}^{2}\right]^{\frac{1}{2}}$ is shown in Fig. 6. The data display a clear increase of $\sigma$ with lowering the temperature due to the term $\sigma_{\mathrm{sc}}(T)=\gamma_{\mu} \sqrt{\Delta B^{2}}$, which expresses the inhomogeneous field distribution associated with the formation of the vortex state in superconducting FeSe below $\sim 60 \mathrm{~K} . \sigma_{\mathrm{nm}}\left(\approx_{\sigma_{\mathrm{ZF}}}\right)$ is caused by the dipolar field contribution of the nuclear moments and is temperature independent. The average spin precession frequency, which is proportional to the average local field, corresponds very closely to the applied field as expected from a demagnetizing factor close to 1 in our geometry. Our ex situ value of $T_{c}$ agrees well with the temperature for gap opening observed in several in situ ARPES measurements [9-13]. The measurement at $5 \mathrm{~K}$ of $\sigma$ as a function of depth by varying the muon implantation energy $E$ further establishes the source of the observed superconductivity. As expected from the TRIM.SP calculations, we observe the largest field inhomogeneity at $\sim 3$ $\mathrm{keV}$, where most of the muons are implanted very close to the FeSe layers. $\sigma_{\mathrm{nm}}$ is small and temperature independent but slightly depends on the muon implantation energy due to the different nuclear moment contribution in the various

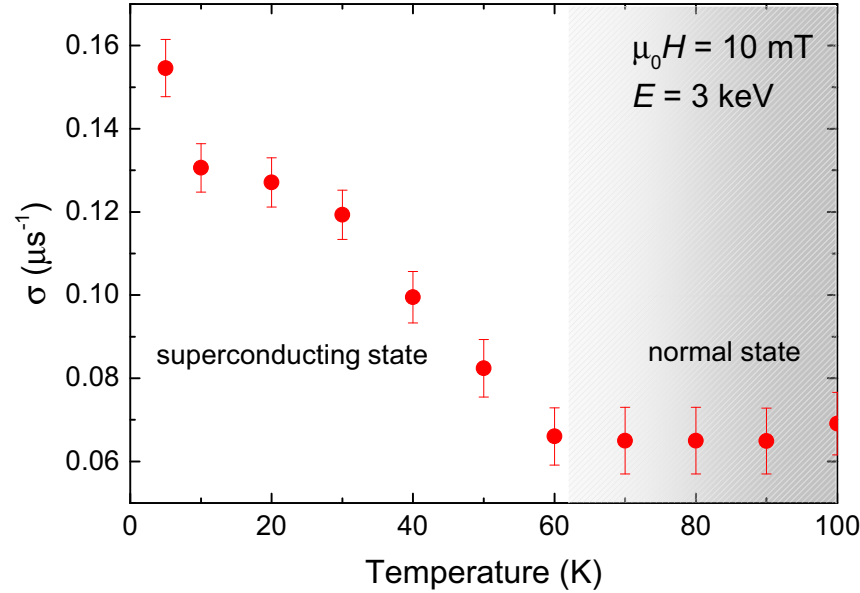

FIG. 6. Temperature dependence of the muon spin damping rate. The temperature dependence of the muon spin damping rate $\sigma$ measured at an implantation energy of $3 \mathrm{keV}$ and an applied field of $10 \mathrm{mT}$.

layers composing the heterostructure. We determined this contribution by performing a full energy scan in the normal state at $T=100 \mathrm{~K}$ and corrected for it to obtain the energy dependence of the field broadening $\sigma_{\mathrm{sc}}$ in the vortex state of FeSe (Fig. 7).

\section{B. Calculation of the field width}

$\mu \mathrm{SR}$ has been widely used to characterize the properties of bulk superconductors and determine their microscopic parameters [39]. For a bulk superconductor in the vortex state, the field broadening is directly given by the magnetic penetration depth $\sigma_{\mathrm{sc}} \propto \frac{1}{\lambda^{2}}$. In our sample, $\sigma_{\mathrm{sc}}(T)$ is determined by the $2 \mathrm{D}$ pancakelike vortices that form in a thin superconducting layer [40,41]. Since the muon stopping profile encompasses a region outside the single FeSe layer (see Fig. 3), the inhomogeneous stray field of the vortices, which extends outside the superconducting layer $[41,42]$, has to be taken into account to obtain

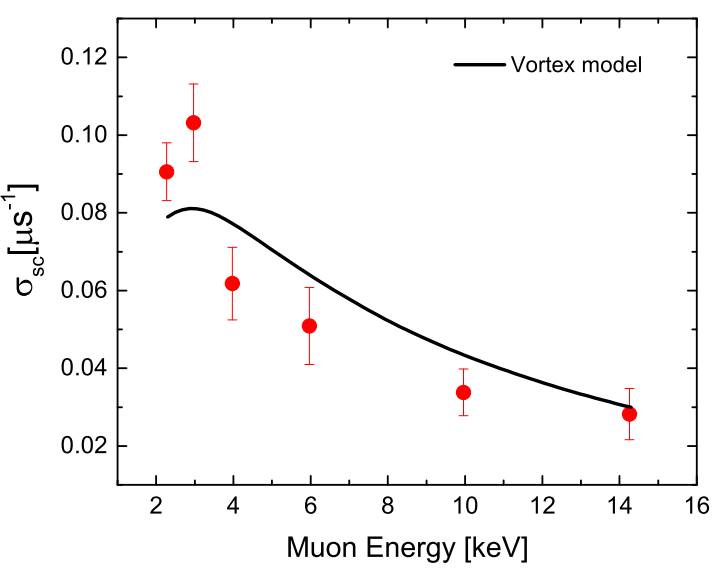

FIG. 7. Energy dependence of the field broadening. Muon spin damping rate $\sigma_{\mathrm{sc}}$ after correction of the nuclear moments contribution plotted as a function of muon implantation energy $E, 5 \mathrm{~K}$. The solid line shows the fit with the $\sigma_{\mathrm{sc}}$ vs $E$ curve calculated within the London model of a very thin superconducting layer as described in the text. 
the relationship between $\sigma_{\mathrm{sc}}(T)$ and the effective superfluid density in FeSe.

The field profile and distribution have been obtained by solving the London equation, which is appropriate for an extreme type-II superconductor $(\xi \ll \lambda$, $\xi$ coherence length $\sim 2-3 \mathrm{~nm}$ [17]). For the ultrathin FeSe layers, application of a magnetic field will lead to the formation of a regular vortex structure of hexagonal symmetry, with each vortex carrying a flux quantum $\Phi_{0}$ and intervortex separation $D \equiv$ $\sqrt{2 \frac{\Phi_{0}}{\sqrt{3} B_{0}}} \cong 490 \mathrm{~nm}$ for $B_{0}=10 \mathrm{mT}$. Indication of such a structure has been visualized by STM measurements [24]. In a bulk superconductor, the local field $B_{z}(x, y, z)$, although varying with the planar coordinates $x$ and $y$, is always parallel to the applied field and perpendicular to the sample surface ( $z$ direction, $z=0$ center of the single layer). In our case, near the single layer, the field lines splay out. However, this effect on the $\mu \mathrm{SR}$ signal is small and we can consider the normal component of the field [41].

We determine $B_{z}(x, y, z)$ from the requirement that it fulfils London equation with source terms representing the flux lines core in a very thin superconducting film $(-d / 2<z<d / 2)$ and Laplace equation outside

$$
-\nabla^{2} B_{z}(x, y, z)+\Pi(z) \frac{B_{z}(x, y, z)}{\lambda^{2}}=\Pi(z) \frac{\Phi_{0}}{\lambda^{2}} \sum_{\vec{R}} \delta(\vec{r}-\vec{R}),
$$

where $\Pi(z)$ is the boxcar function, which is equal to 1 for $-d / 2 \leqslant z \leqslant d / 2$ and 0 otherwise, $\vec{r}=(x, y)$, and $\vec{R}$ the vortex positions. The solution is obtained by decomposing $B_{z}(x, y, z)$ into its Fourier components in the $x-y$ plane

$$
B_{z}(x, y, z)=\sum_{\vec{k}} b_{z}(\vec{k}, z) e^{-i \vec{k} \cdot \vec{r}}
$$

where $\vec{k}$ is the reciprocal lattice vector of the flux lattice with $k=|\vec{k}|=\sqrt{\frac{16 \pi^{2}\left(m^{2}-m n+n^{2}\right)}{3 D^{2}}}, m, n$ integer. After matching the field and its derivative at the layer boundaries, we determine the Fourier coefficients $b_{z}(\vec{k}, z)$ so that solutions are obtained inside and outside the single-layer FeSe. The width of the field distribution at $z$ is then given by $\Delta B_{z}^{2}(z)=\left\langle B_{z}^{2}(z)\right\rangle-\left\langle B_{z}(z)\right\rangle^{2}=$ $\sum_{k \neq 0} b_{z}(k, z)^{2}$. Averaging is over the $x$ and $y$ plane coordinates. For a comparison with the measured broadening, $\Delta B_{z}(z)$ has to be weighted with the normalized muon stopping distribution $n(z, E)$ so that $\sigma_{\mathrm{sc}}^{2}(E)=\gamma_{\mu}^{2} \int_{-\infty}^{\infty} \Delta B_{z}^{2}(z) n(z, E) d z$. In contrast to the $3 \mathrm{D}$ case where $\sigma_{\mathrm{sc}} \propto \frac{1}{\lambda^{2}}$, in our $2 \mathrm{D}$ situation we find that the field broadening is governed by the Pearl length scale $\Lambda_{P} \equiv 2 \lambda^{2} / d$ as expected for the vortex state in superconducting films with $d \ll \lambda$ [43]. For instance, taking into account that the superconducting layer is very thin and that the dominating contribution to the observed field broadening comes from the muons stopping outside the layer $(d / 2 \leqslant z \leqslant$ $-d / 2$ ), one finds that the Fourier coefficients can be expressed as $b_{z}(k, z) \cong \frac{B_{\text {appl }}}{\Lambda_{P}} \frac{e^{-i k|z|}}{k}$.

\section{Determination of microscopic superconducting properties}

The Pearl length scale is directly related to the sheet superconducting carrier density $n_{s}^{2 \mathrm{D}}=\frac{2 m_{e}^{*}}{\mu_{0} e^{2} \Lambda_{P}}$. Figure 8 shows

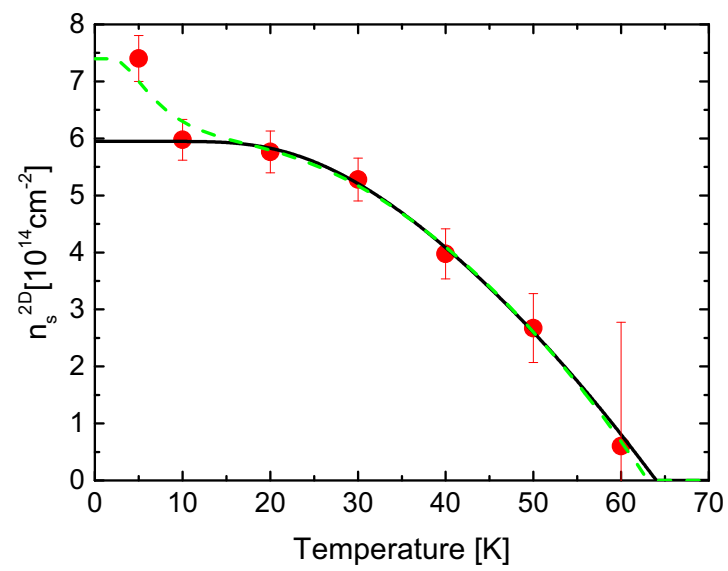

FIG. 8. Temperature dependence of the superfluid sheet density. Superfluid 2D density versus temperature for ultrathin FeSe. The solid curve is a fit with a BCS $s$-wave gap. For comparison, a model assuming an additional small gap manifesting itself at low temperatures is shown as a dashed line.

the temperature dependence of the sheet superfluid density in the ultrathin FeSe layer. Remarkably, $n_{s}^{2 \mathrm{D}}$ does not show any signs of phase fluctuations, which may be expected in a 2D-like superconductor, probably because of the strong coupling to the STO substrate [10]. This temperature dependence can be well fitted down to $10 \mathrm{~K}$ using a single-gap BCS $s$-wave model (solid line in Fig. 8). The fit gives a gap value at zero temperature $\Delta(0)=10.2(1.1) \mathrm{meV}$ and $T_{c}=62(2) \mathrm{K}$. This gap value is consistent with several ARPES and STM measurements that find values in the range 10-15 meV $[10-13,44]$. Some STM measurements have reported gap structures with double peaks at $\approx 10 \mathrm{meV}$ and 15-20 meV [9]. These differences may be due to differences in annealing conditions of the sample, protection layer, or substrate preparation [45]. It is worth noting that since the muons uniformly probe the entire area of the sample, the measured parameters are sample average values, which may explain why our gap value is on the lower side of ARPES and STM values. $\mu$ SR is able to discriminate between different electronic phases. Our TF- $\mu$ SR data can be fitted with a single superconducting component. Therefore, the results show that homogeneous superconductivity exists across the entire FeSe/STO interface of size $\sim \mathrm{cm}^{2}$. By homogeneity, we mean here (a) homogeneous superconductivity on a scale of the order of the Pearl length scale or larger and (b) that on this scale there is no phase separation, e.g., in superconducting and nonsuperconducting regions. This does not exclude, however, local inhomogeneity at nanoscale or subnanoscale. The gap to $T_{c}$ ratio $\frac{\Delta(0)}{k_{B} T_{c}}=1.9(2)$ puts the single-layer FeSe in the category of the moderately strong-coupling superconductors.

Fitting the measured energy dependence of $\sigma_{\mathrm{sc}}(E)$ (Fig. 7) with our model, we obtain $\Lambda_{P}=2.49(5) \times 10^{4} \mathrm{~nm}$ at $10 \mathrm{~K}$. From this number we estimate the density of paired electrons to be $n_{s}^{2 \mathrm{D}} \simeq 6 \times 10^{14} \mathrm{~cm}^{-2}$. For this estimate we take $m^{*}=$ $2.7 m_{e}$ as determined by ARPES measurements [11], which are based on a parabolic fitting of the band dispersion, a good approximation for FeSe [14]. Moreover, such a band dispersion is also implicit in the BCS model that we use to determine the superconducting parameters [Eq. (6)]. The 
choice of $m^{*}=2.7 m_{e}$ is also confirmed by recent measurement of electron-doped FeSe [21], where the effective mass of the electron band at $M$ (relevant band in single layer FeSe) was found to lie between $2.7 m_{e}$ and $3.5 m_{e}$ (for a doping of 0.1-0.12 electrons per $\mathrm{Fe}$, which corresponds to the electron excess value in single-layer FeSe). Since $n_{s}^{2 \mathrm{D}} \propto \frac{m^{*}}{\Lambda_{P}}$, a value of $2.7 m_{e}$ sets rather a lower limit for $n_{s}^{2 \mathrm{D}}$. On the other hand, disorder of the vortex lattice would increase the value of $\Lambda_{P}$. However, disorder contributes only quadratically to the measured spin relaxation rate so that even a contribution equal to the broadening associated with the vortex field would decrease $n_{s}^{2 \mathrm{D}}$ by $\sim 40 \%$. Overall, we assign an error to our estimate of the sheet carrier density of $\pm 30 \%$.

Not many methods are able to determine the superconducting carrier density of very thin layers. More important, we would like to stress here that unlike other techniques our measurement provides a direct estimate of the paired carriers in a buried FeSe layer. The determination of this quantity is of relevance, for instance, to clarify its link to the enhancement of superconductivity with respect to the bulk counterpart and to understand the mechanism working at the interface between the FeSe layer and the substrate. Charge (electron) doping by ionic liquid gating $[18,19]$ or from deposited $\mathrm{K}$ atoms has been found important on its own to raise $T_{c}$. On the other hand, ARPES spectroscopy studies indicate that, beyond that, interface coupling may be necessary to get the highest $T_{c}$ close to liquid $\mathrm{N}_{2}$ temperature [14]. Assuming a dominant electron character, a Hall measurement (Hall coefficient $R_{H}=\frac{1}{n_{e} e} \simeq$ $-3 \times 10^{-3} \mathrm{~cm}^{-3} / \mathrm{C}$ ) of thin ionic liquid gated FeSe flakes [18] with $T_{c} \sim 48 \mathrm{~K}$ gives a carrier density at $50 \mathrm{~K}$ of $2 \times$ $10^{21} \mathrm{~cm}^{-3}$. ARPES measurements of the electronic structure of single-layer FeSe with $T_{c} \approx 60 \mathrm{~K}$ estimate an electron counting of $\sim 0.12$ electron/Fe [11,13], which corresponds to a similar volume density of carrier $2.2 \times 10^{21} \mathrm{~cm}^{-3}$ in a $0.6-$ $\mathrm{nm}$-thick monolayer. By contrast, other transport experiments have reported much higher numbers of Hall carriers. From $R_{H} \simeq-0.33 \times 10^{-3} \mathrm{~cm}^{-3} / \mathrm{C}$, a value $n_{e} \simeq 1.9 \times 10^{22} \mathrm{~cm}^{-3}$ has been inferred for 2.9-nm-thick FeSe on $\mathrm{MgO}$ at $50 \mathrm{~K}$ [19]. Similar high values have been found for one monolayer FeSe/STO capped by FeTe/Si [45], indicating that the above-mentioned agreement of transport and ARPES carrier determination may be fortuitous. However, multiband effects and different types of carrier make it difficult to determine the relevant carrier density from the $R_{H}$ value, which is strongly dependent on temperature and growth/annealing conditions [45] and may not give a reliable measure of the actual number of carriers that condense in the superconducting state.

A related question is the spatial extent of superconductivity in FeSe layers more than one monolayer thick. Although spectroscopic data indicate that additional layers have weak interlayer coupling with the second monolayer displaying semiconducting characteristics and charge neutrality [13], the question of the contribution of additional layers to the superconductivity of $1 \mathrm{ML} \mathrm{FeSe}$ is not fully understood. Shiogai et al. [17,19] used an electric double-layer transistor configuration, which allows at the same time electrostatic carrier doping and electrochemical thickness tuning, to identify a unified trend of $T_{c}$ vs $R_{H}$ for ultrathin FeSe layers on oxide substrates such as $\mathrm{SrTiO}_{3}, \mathrm{MgO}$, and $\mathrm{KTaO}_{3}$ and determine various length scales and critical thicknesses. Particularly, Hall measurements as a function of thickness allowed to determine the length scale of the charge distribution due to charge transfer from the substrate, $d_{\mathrm{CT}}$, and the penetration length of the superconducting order parameter $\xi_{N}^{\mathrm{CT}}$ in the layer above due to the proximity effect. For FeSe/STO, $d_{\mathrm{CT}} \cong$ $4 \mathrm{~nm}$ and $\xi_{\mathrm{N}}^{\mathrm{CT}} \cong 3.5 \mathrm{~nm}$, implying that ultrathin FeSe may exhibit high- $T_{c}$ superconductivity on an effective length higher than that inferred by ARPES measurements of the electronic structure of $\geqslant 2 \mathrm{ML}$ FeSe. Even allowing for band-bending effects increasing the thickness of the charge transfer layer in the specific electric dipole layer configuration of Ref. [19], it appears reasonable to consider that proximity effects cannot be ignored in $>1 \mathrm{ML}$ thick FeSe layer. In this respect, it is interesting to note that the value $n_{s}^{2 \mathrm{D}} \simeq 1.4 \times 10^{14} \mathrm{~cm}^{-2}$ obtained from the excess electron determination by ARPES is about a factor of 4 lower than the present determination of the superconducting carrier density $n_{s}^{2 \mathrm{D}} \simeq 6 \pm 2 \times 10^{14} \mathrm{~cm}^{-2}$ of our heterostructure containing $1+4 \mathrm{FeSe}$ layers.

The temperature dependence of the superfluid density (Fig. 8) may suggest an increase of this quantity at the lowest measured temperature, $5 \mathrm{~K}$. Since this effect appears only in a single data point, we can only speculate about its significance. It might point to the presence of a second (small) gap effectively opening below $10 \mathrm{~K}$. We tried a two-gap $(s+s)$-wave model to account for this low-temperature increase. For this we analyzed our data with a phenomenological model by assuming two independent contributions to the total superfluid density but with a common $T_{c}$. The functional form of the two-gap model, which includes as a special case the single-gap model, previously discussed, is [46]

$$
\frac{n_{s}^{2 \mathrm{D}}(T)}{n_{s}^{2 \mathrm{D}}(0)}=\omega \frac{n_{s}^{2 \mathrm{D}}\left(T, \Delta_{1}(0)\right)}{n_{s}^{2 \mathrm{D}}\left(0, \Delta_{1}(0)\right)}+(1-\omega) \frac{n_{s}^{2 \mathrm{D}}\left(T, \Delta_{2}(0)\right)}{n_{s}^{2 \mathrm{D}}\left(0, \Delta_{2}(0)\right)},
$$

where $\lambda(0)$ is the value of the penetration depth at $T=0 \mathrm{~K}$, $\Delta_{\mathrm{i}}(0)$ is the value of the $i$ th $(i=1$ or 2$)$ superconducting gap at $T=0 \mathrm{~K}$, and $\omega$ is the weighting factor of the band with the largest gap.

Each component of Eq. (5) can be calculated within the local London approximation $(\lambda \gg \xi)[47,48]$ as

$$
\frac{n_{s}^{2 \mathrm{D}}\left(T, \Delta_{\mathrm{i}}(0)\right)}{n_{s}^{2 \mathrm{D}}\left(0, \Delta_{\mathrm{i}}(0)\right)}=1+2 \int_{\Delta_{\mathrm{i}}(0)}^{\infty}\left(\frac{\partial f}{\partial E}\right) \frac{E d E}{\sqrt{E^{2}-\Delta_{\mathrm{i}}(T)^{2}}}
$$

where $f=\left[1+\exp \left(E / k_{\mathrm{B}} T\right)\right]^{-1}$ is the Fermi function, and $\Delta_{\mathrm{i}}(T)=\Delta_{\mathrm{i}}(0) \delta\left(T / T_{\mathrm{c}}\right)$. The temperature dependence of the gap is parametrized by the expression $\delta\left(T / T_{\mathrm{c}}\right)=$ $\tanh \left\{1.82\left[1.018\left(T_{\mathrm{c}} / T-1\right)\right]^{0.51}\right\}$, which well represents the temperature dependence of a BCS gap [49].

A fit is shown as dashed line in Fig. 8 yielding for the main gap $\Delta(0)=10.5(1.6) \mathrm{meV}$ (in agreement with the singlegap fit) and the putative small gap $\Delta(0)=1.3(6) \mathrm{meV}$ with relative weight $0.23(4)$. It is also interesting to note that a similar upturn in the superfluid density has been observed in intercalated FeSe [50] in which the FeSe layers are well separated by an intercalating layer, possibly approaching the 2D limit of this work. Another possibility may be some proximity contribution of the additional 4 monolayers of FeSe 
modifying the gap structure. Further measurements are needed to elucidate these points, as well as the question about the possible presence of additional small gaps at much lower temperature and their nodal structure.

To conclude, by measuring ex situ the depth and temperature dependence of the local field distribution in a heterostructure containing a buried superconducting ultrathin FeSe layer, we detect the formation of a vortex state below $T_{c} \cong 60 \mathrm{~K}$ and quantify the superfluid density of $(1+4) \mathrm{ML}$ FeSe. The temperature dependence can be well explained by a single BCS $s$-wave gap of $10.2(1.1) \mathrm{meV}$. The $\mu \mathrm{SR}$ spectra show that the vortex state and superconductivity are homogeneously formed across the entire interface over a sample with a sizable amount of charges condensing below $T_{c} \approx 62 \mathrm{~K}$. This shows that superconductivity in the buried interface has stable character and that inhomogeneities or imperfections of the substrate or of the overlayers do not hamper the formation of a superconducting state nor sizably modify its properties. A very sensitive magnetic probe such as polarized muons does not see indication of static or dynamic magnetism. The simple structure of single-layer FeSe, its high $T_{c}$ with $s$-wave type of gap, and rather clean BCS character make it an ideal system to develop a microscopic understanding of high- $T_{c}$ superconductivity.
[1] J. G. Bednorz and K. A. Müller, Possible high-T superconductivity in Ba-La-Cu-O system, Z. Phys. D 64, 189 (1986).

[2] A. Schilling, M. Cantoni, J. D. Guo, and H. R. Ott, Superconductivity above $130 \mathrm{~K}$ in the $\mathrm{Hg}-\mathrm{Ba}-\mathrm{Ca}-\mathrm{Cu}-\mathrm{O}$ system, Nature (London) 363, 56 (1993).

[3] Y. Kamihara, T. Watanabe, M. Hirano, and H. Hosono, IronBased Layered Superconductor $\mathrm{La}\left[\mathrm{O}_{1-x} \mathrm{~F}_{x}\right] \mathrm{FeAs}(x=0.05-$ 0.12) with $T_{c}=26 \mathrm{~K}$, J. Am. Chem. Soc. 130, 3296 (2008).

[4] F.-C. Hsu, J.-Y. Luo, K.-W. Yeh, T.-K. Chen, T.-W. Huang, P. M. Wu, Y.-C. Lee, Y.-L. Huang, Y.-Y. Chu, D.-C. Yan et al., Superconductivity in the PbO-type structure $\alpha$-FeSe, Proc. Natl. Acad. Sci. USA 105, 14262 (2008).

[5] D. C. Johnston, The puzzle of high temperature superconductivity in layered iron pnictides and chalcogenides, Adv. Phys. 59, 803 (2010).

[6] J. Paglione and R. L. Greene, High-temperature superconductivity in iron-based materials, Nat. Phys. 6, 645 (2010).

[7] G. R. Stewart, Superconductivity in iron compounds, Rev. Mod. Phys. 83, 1589 (2011).

[8] F. Wang and D. H. Lee, The electron-pairing mechanism of ironbased superconductors, Science 332, 200 (2011).

[9] Q. Y. Wang, Z. Li, W. H. Zhang, Z. C. Zhang, J.-S. Zhang, W. Li, H. Ding, Y.-B. Ou, P. Deng, K. Chang et al., Interface-Induced High-Temperature Superconductivity in Single Unit-Cell FeSe Films on $\mathrm{SrTiO}_{3}$, Chin. Phys. Lett. 29, 037402 (2012).

[10] J. J. Lee, F. T. Schmitt, R. G. Moore, S. Johnston, Y. T. Cui, W. Li, M. Yi, Z. K. Liu, M. Hashimoto et al., Interfacial mode coupling as the origin of the enhancement of $T_{c}$ in FeSe films on $\mathrm{SrTiO}_{3}$, Nature (London) 515, 245 (2014).

[11] D. F. Liu, W. H. Zhang, D. X. Mou, J. F. He, Y. B. Ou, Q. Y. Wang, Z. Li, L. L. Wang, L. Zhao, S. L. He et al., Electronic origin of high-temperature superconductivity in single-layer FeSe superconductor, Nat. Commun. 3, 931 (2012).

[12] S. L. He, J. F. He, W. H. Zhang, L. Zhao, D. F. Liu, X. Liu, D. X. Mou, Y. B. Ou, Q. Y. Wang, Z. Li et al., Phase diagram and electronic indication of high-temperature superconductivity at $65 \mathrm{~K}$ in single-layer FeSe films, Nat. Mater. 12, 605 (2013).

[13] S. Y. Tan, Y. Zhang, M. Xia, Z. R. Ye, F. Chen, X. Xie, R. Peng, D. F. Xu, Q. Fan, H. C. Xu et al., Interface-induced superconductivity and strain-dependent spin density waves in $\mathrm{FeSe} / \mathrm{SrTiO}_{3}$ thin films, Nat. Mater. 12, 634 (2013).

[14] S. N. Rebec, T. Jia, C. Zhang, M. Hashimoto, D.-H. Lu, R. G. Moore, and Z.-X. Shen, Coexistence of Replica Bands and
Superconductivity in FeSe Monolayer Films, Phys. Rev. Lett. 118, 067002 (2017).

[15] W.-H. Zhang, Y. Sun, J.-S. Zhang, F.-S. Li, M.-H. Guo, Y.-F. Zhao, H.-M. Zhang, J.-P. Peng, Y. Xing, H.-C. Wang et al., Direct observation of high-temperature superconductivity in one-unitcell FeSe films, Chin. Phys. Lett. 31, 017401 (2014).

[16] Z. Wang, C. Liu, Y. Liu, and J. Wang, High-temperature superconductivity in one-unit-cell FeSe films, J. Phys.: Condens. Matter 29, 153001 (2017).

[17] J. Shiogai, Y. Ito, T. Mitsuhashi, T. Nojima, and A. Tsukazaki, Electric-field-induced superconductivity in electrochemically etched ultrathin FeSe films on $\mathrm{SrTiO}_{3}$ and $\mathrm{MgO}$, Nat. Phys. 12, 42 (2016).

[18] B. Lei, J. H. Cui, Z. J. Xiang, C. Shang, N. Z. Wang, G. J. Ye, X. G. Luo, T. Wu, Z. Sun, and X. H. Chen, Evolution of HighTemperature Superconductivity from a Low $-T_{c}$ Phase Tuned by Carrier Concentration in FeSe Thin Flakes, Phys. Rev. Lett. 116, 077002 (2016).

[19] J. Shiogai, T. Miyakawa, Y. Ito, T. Nojima, and A Tsukazaki, Unified trend of superconducting transition temperature versus Hall coefficient for ultrathin FeSe films prepared on different oxide substrates, Phys. Rev. B 95, 115101 (2017).

[20] H. Ding, Y.-F. Lv, K. Zhao, W.-L. Wang, L. Wang, C.-L. Song, X. Chen, X.-C. Ma, and Q.-K. Xue, High-Temperature Superconductivity in Single-Unit-Cell FeSe Films on Anatase $\mathrm{TiO}_{2}$ (001), Phys. Rev. Lett. 117, 067001 (2016).

[21] C. H. P. Wen, H. C. Xu, C. Chen, Z. C. Huang, X. Lou, Y. J. Pu, Q. Song, B. P. Xie, Mahmoud Abdel-Hafiez, D. A. Chareev et al., Anomalous correlation effects and unique phase diagram of electron-doped FeSe revealed by photoemission spectroscopy, Nat. Commun. 7, 10840 (2016).

[22] J. F. Ge, Z.-L. Liu, C. Liu, C.-L. Gao, D. Qian, Q.-K. Xue, Y. Liu, and J.-F. Jia, Superconductivity above $100 \mathrm{~K}$ in single-layer FeSe films on doped $\mathrm{SrTiO}_{3}$, Nat. Mater. 14, 285 (2015).

[23] Z. Zhang, Y.-H. Wang, Q. Song, C. Liu, R. Peng, K. A. Moler, D. Feng, and Y. Wang, Onset of the Meissner effect at $65 \mathrm{~K}$ in FeSe thin film grown on $\mathrm{Nb}$-doped $\mathrm{SrTiO}_{3}$ substrate, Sci. Bulletin 60, 1301 (2015).

[24] Q. Fan, W. H. Zhang, X. Liu, Y. J. Yan, M. Q. Ren, R. Peng, H. C. Xu, B. P. Xie, J. P. Hu, T. Zhang et al., Plain $s$-wave superconductivity in single-layer $\mathrm{FeSe}$ on $\mathrm{SrTiO}_{3}$ probed by scanning tunneling microscopy, Nat. Phys. 11, 946 (2015). 
[25] H. Saadaoui, Z. Salman, T. Prokscha, A. Suter, B. M. Wojek, and E. Morenzoni, Zero-field spin depolarization of low-energy muons in ferromagnetic nickel and silver metal, Physics Procedia 30, 164 (2012).

[26] E. Morenzoni, T. Prokscha, A. Suter, H. Luetkens, and R. Khasanov, Nano-scale thin film investigations with slow polarized muons, J. Phys.: Condens. Matter 16, S4583 (2004).

[27] T. Prokscha, E. Morenzoni, K. Deiters, F. Foroughi, D. George, R. Kobler, A. Suter, and V. Vrankovic, The new $\mu$ E4 beam at PSI: A hybrid-type large acceptance channel for the generation of a high intensity surface-muon beam, Nucl. Instrum. Methods Phys. Res., Sect. A 595, 317 (2008).

[28] E. Morenzoni, H. Glückler, T. Prokscha, H. P. Weber, E. M. Forgan, T. J. Jackson, H. Luetkens, Ch. Niedermayer, M. Pleines, M. Birke et al., Low-energy $\mu$ SR at PSI: present and future, Phys. B (Amsterdam) 289, 653 (2000).

[29] Ch. Niedermayer, E. M. Forgan, H. Glückler, A. Hofer, E. Morenzoni, M. Pleines, T. Prokscha, T. M. Riseman, M. Birke, T. J. Jackson, Ch. Niedermayer et al., Direct Observation of a Flux Line Lattice Field Distribution Across an $\mathrm{YBa}_{2} \mathrm{Cu}_{3} \mathrm{O}_{7-\delta}$ Surface by Low Energy Muons, Phys. Rev. Lett. 83, 3932 (1999).

[30] E. Morenzoni, B. M. Wojek, A. Suter, T. Prokscha, G. Logvenov, and I. Božović, The Meissner effect in a strongly underdoped cuprate above its critical temperature, Nat. Commun. 2, 272 (2011).

[31] A. Di Bernardo, Z. Salman, X. L. Wang, M. Amado, M. Egilmez, M. G. Flokstra, A. Suter, S. L. Lee, J. H. Zhao, T. Prokscha et al., Intrinsic Paramagnetic Meissner Effect Due to $s$-Wave OddFrequency Superconductivity, Phys. Rev. X 5, 041021 (2015).

[32] F. Al Ma'Mari, T. Moorsom, G. Teobaldi, W. Deacon, T. Prokscha, H. Luetkens, S. Lee, G. E. Sterbinsky, D. A. Arena, D. A. MacLaren et al., Beating the Stoner criterion using molecular interfaces, Nature (London) 524, 69 (2015).

[33] W. Eckstein, Computer Simulations of Ion-Solid Interactions (Springer, Berlin, 1991).

[34] E. Morenzoni, H. Glückler, T. Prokscha, R. Khasanov, H. Luetkens, M. Birke, E.M. Forgan, Ch. Niedermayer, and M. Pleines, Implantation studies of $\mathrm{keV}$ positive muons in thin metallic layers, Nucl. Instrum. Methods 192, 254 (2002).

[35] G. M. Luke, Y. Fudamoto, K. M. Kojima, M. I. Larkin, J. Merrin, B. Nachumi, Y. J. Uemura, Y. Maeno, Z. Q. Mao, Y. Mori et al., Time-reversal symmetry breaking superconductivity in $\mathrm{Sr}_{2} \mathrm{RuO}_{4}$, Nature (London) 394, 558 (1998).
[36] P. K. Biswas, H. Luetkens, T. Neupert, T. Stürzer, C. Baines, G. Pascua, A. P. Schnyder, M. H. Fischer, J. Goryo, M. R. Lees et al., Evidence for superconductivity with broken time-reversal symmetry in locally noncentrosymmetric SrPtAs, Phys. Rev. B 87, 180503(R) (2013).

[37] R. A. Kubo, stochastic theory of spin relaxation, Hyperfine Interact. 8, 731 (1981).

[38] A. Suter and B. M. Wojek, Musrfit: a free platform-independent framework for $\mu$ SR data analysis, Phys. Procedia 30, 69 (2012).

[39] J. E. Sonier, J. H. Brewer, and R. F. Kiefl, $\mu$ SR studies of the vortex state in type-II superconductors, Rev. Mod. Phys. 72, 769 (2000).

[40] J. R. Clem, Two-dimensional vortices in a stack of thin superconducting films: A model for high-temperature superconducting multilayers, Phys. Rev. B 43, 7837 (1991).

[41] E. H. Brandt, Ginzburg-Landau vortex lattice in superconductor films of finite thickness, Phys. Rev. B 71, 014521 (2005).

[42] G. Carneiro and E. H. Brandt, Vortex lines in films: Fields and interactions, Phys. Rev. B 61, 6370 (2000).

[43] J. Pearl, Current distribution in superconducting films carrying quantized fluxoids, Appl. Phys. Lett. 5, 65 (1964).

[44] D. Huang, C.-L. Song, T. A. Webb, S. Fang, C.-Z. Chang, J. S Moodera, E. Kaxiras, and J. E. Hoffman, Revealing the EmptyState Electronic Structure of Single-Unit-Cell FeSe/ $\mathrm{SrTiO}_{3}$, Phys. Rev. Lett. 115, 017002 (2015)

[45] W. Zhang et al., Interface charge doping effects on superconductivity of single-unit-cell FeSe films on $\mathrm{SrTiO}_{3}$ substrates, Phys. Rev. B 89, 060506(R) (2014).

[46] H. Padamsee, J. E. Neighbor, and C. A. Shiffman, Quasiparticle phenomenology for thermodynamics of strong-coupling superconductors, J. Low Temp. Phys. 12, 387 (1973).

[47] M. Tinkham, Introduction to Superconductivity (McGraw-Hill, New York, 1975)

[48] R. Prozorov and R. W. Giannetta, Magnetic penetration depth in unconventional superconductors, Supercond. Sci. Technol. 19, R41 (2006).

[49] A. Carrington and F. Manzano, Magnetic penetration depth of $\mathrm{MgB}_{2}$, Phys. C (Amsterdam) 385, 205 (2003).

[50] M. Burrard-Lucas, D. G. Free, S. J. Sedlmaier, J. D. Wright, S. J. Cassidy, Y. Hara, A. J. Corkett, T. Lancaster, P. J. Baker, S. J. Blundell et al., Enhancement of the superconducting transition temperature of FeSe by intercalation of a molecular spacer layer, Nat. Mater. 12, 15 (2013). 